\title{
Viral metagenomics of six bat species in close contact with humans in southern China
}

\author{
Xue-yan Zheng ${ }^{1,2} \cdot$ Min Qiu ${ }^{1}$ Wei-jie Guan ${ }^{3} \cdot$ Jin-ming $\mathrm{Li}^{4} \cdot$ Shao-wei Chen ${ }^{1} \cdot$ \\ Ming-ji Cheng ${ }^{1} \cdot$ Shu-ting Huo ${ }^{1} \cdot$ Zhong Chen $^{5} \cdot$ Yi Wu $^{6} \cdot$ Li-na Jiang ${ }^{1} \cdot$ Qing Chen $^{1}$
}

Received: 16 January 2017 / Accepted: 14 September 2017 / Published online: 5 October 2017

(C) Springer-Verlag GmbH Austria 2017

\begin{abstract}
Accumulating studies have shown that bats could harbor various important pathogenic viruses that could be transmitted to humans and other animals. Extensive metagenomic studies of different organs/tissues from bats have revealed a large number of novel or divergent viruses. To elucidate viral diversity and epidemiological and phylogenetic characteristics, six pooled fecal samples from bats were generated (based on bat species and geographic regions characteristic for virome analysis). These contained 500 fecal samples from six bat species, collected
\end{abstract}

Handling Editor: Zhongjie Shi.

Electronic supplementary material The online version of this article (doi:10.1007/s00705-017-3570-3) contains supplementary material, which is available to authorized users.

Qing Chen

qch.2009@163.com

1 Department of Epidemiology, School of Public Health, Southern Medical University, 1838 Guangzhou Avenue North, Guangzhou, Guangdong, China

2 Institute of Non-communicable Disease Control and Prevention, Guangdong Provincial Center for Disease Control and Prevention, Guangzhou, China

3 State Key Laboratory of Respiratory Disease, National Clinical Research Center for Respiratory Disease, Guangzhou Institute of Respiratory Disease, Guangzhou, Guangdong, China

4 Department of Bioinformatics, School of Basic Medical Sciences, Southern Medical University, Guangzhou, Guangdong, China

5 College of Life Science, Hainan Normal University, Haikou, Hainan, China

6 College of Life Science, Guangzhou University, Guangzhou, Guangdong, China in four geographic regions. Metagenomic analysis revealed a plethora of divergent viruses originally found in bats. Multiple contigs from influenza A virus and coronaviruses in bats shared high identity with those from humans, suggesting possible cross-species transmission, whereas a number of contigs, whose sequences were taxonomically classifiable within Alphapapillomavirus, Betaretrovirus, Alpharetrovirus, Varicellovirus, Cyprinivirus, Chlorovirus and $\mathrm{Cucu-}$ movirus had low identity to viruses in existing databases, which indicated possible evolution of novel viral species. None of the established caliciviruses and picornaviruses were found in the 500 fecal specimens. Papillomaviruses with high amino acid identity were found in Scotophilus kuhlii and Rhinolophus blythi, challenging the hypotheses regarding the strict host specificity and co-evolution of papillomaviruses. Phylogenetic analysis showed that four bat rotavirus A strains might be tentative G3 strains, according to the Rotavirus Classification Working Group classification.

\section{Introduction}

As members of the order Chiroptera, the second largest group ( 25\%) of mammalian species [1], more than 1,240 bat species are currently found worldwide, everywhere except the South and North Poles. Recent studies have successfully identified novel bat viruses on the basis of consensus primers or sequence-independent PCR amplification [2-4]. Over 80 virus species have been detected in bats, including several emerging human pathogens (i.e., severe acute respiratory syndrome coronaviruses, lyssaviruses, henipaviruses, Marburg virus and Ebola virus) [5]. Furthermore, some bat species live in close proximity to human habitation, highlighting the potential role of bats as reservoirs of zoonotic diseases [6]. 
Using polymerase chain reaction (PCR) screening with consensus primers, closely related viral species in bats have been detected [2-4]. However, the high assay specificity of these methods has limited the detection of divergent or unknown viruses. The advent of next-generation sequencing (NGS) technology has facilitated metagenomic analyses to simultaneously screen for all viral families. NGS has been successfully applied to detect novel viruses in different types of samples (including lungs, liver, brain, pharyngeal swabs and anal swabs) [7] and is expected to provide more in-depth information on previously unidentified viruses and viral taxonomy. Studies from the US [8, 9], France [10], New Zealand [11] and China [12-15] have analyzed the virome profiles of bats with NGS technology and have identified various unknown mammalian viruses. Most of these studies analyzed viral contigs by homogenizing different organs and tissues of bats; however, this approach is restricted by the unclear tissue distribution and route of transmission of bat viruses. The paucity of knowledge on bat-derived viruses warrants more extensive investigation and surveillance of different bat species in various geographic areas. The digestive tract is connected to the outer environment through ingestion of foods and liquids, providing a niche for virus transmission from bats to humans and other animals. Investigation of the virome in fecal samples from bats, and subsequent epidemiological and phylogenetic characterization, of any viruses, would significantly advance our understanding, especially with regards to prevention and management of zoonotic diseases in different geographic regions.

Papillomaviruses (PVs), rotavirus A (RVA), caliciviruses (CVs) and picornaviruses (PiVs) are widespread pathogens causing human diseases and have been detected in fecal samples of some bat species [16-19]. However, it is still unknown whether bats play a role as reservoirs in transmission of these viruses. More extensive studies of the prevalence and characteristics of these viruses isolated from different bat species and in different geographic areas is needed. To conduct this analysis, fecal specimens from six bat species, Cynopterus sphinx, Miniopterus schreibersii, Rousettus leschenaultii, Hipposideros larvatus, Rhinolophus blythi and Scotophilus kuhlii, were collected.

In this study, we report on the virome profile of fecal samples from six bat species in four cities in southern China, using metagenomic analysis, sequence-independent amplification and high-throughput sequencing (Solexa, Illumina).

\section{Materials and methods}

\section{Ethics statement}

The study protocol was reviewed and approved by the Animal Ethics and Welfare Committee of the School of Public
Health and Tropical Medicine, Southern Medical University, China. All animals were treated in strict accordance with the guidelines for Laboratory Animal Use and Care from Southern Medical University and the Rules for the Implementation of Laboratory Animal Medicine (1998) from the Ministry of Health, China.

\section{Sample collection}

Fecal sample collection from bats was performed in Hainan province [Haikou city (latitude: $20.02^{\circ} \mathrm{N}$; longitude: $110.35^{\circ}$ E)], Guangdong province [Huizhou (latitude: $23.11^{\circ} \mathrm{N}$; longitude: $114.42^{\circ} \mathrm{E}$ ), Guangzhou (latitude: $23.13^{\circ} \mathrm{N}$; longitude: $113.26^{\circ} \mathrm{E}$ ) and Yunfu (latitude: $22.92^{\circ} \mathrm{N}$; longitude: $112.04^{\circ} \mathrm{E}$ ) cities], all in southern China. Briefly, fecal samples from bats were immersed into maintenance medium in a virus sampling tube and temporarily stored at $-20{ }^{\circ} \mathrm{C}$. After completion of sampling, fecal samples were transported to the laboratory and stored at $-80{ }^{\circ} \mathrm{C}$. Bat species identification was confirmed by amplification and sequencing of the cytochrome B (cyt $B$ ) gene, which has been commonly applied in archaeology [20].

\section{Purification of fecal samples and extraction of viral nucleic acids}

Tubes with fecal samples in maintenance medium were vigorously vortexed to homogenize the samples. Samples of each species from the same site were then pooled by adding $1 \mathrm{ml}$ from each maintenance medium sample into a fresh sample tube. Six pooled samples, classified by species and collection site, were then centrifuged twice at $13,000 \times \mathrm{g}$ for $20 \mathrm{~min}$ at $4{ }^{\circ} \mathrm{C}$. The supernatant was then filtered through a $0.22 \mu \mathrm{m}$ filter (Millipore Inc., USA) twice to remove eukaryotic cell- and bacterium-sized particles. Filtrates were concentrated in a $100-\mathrm{kDa}$ Pellicon II filter (Millipore Inc., USA). To remove the remaining extra-cellular nucleic acids, the filtrates were treated with DNaseI (NEB, USA) and RNaseIf (NEB). $375 \mu \mathrm{l}$ of the filtrate from each pooled sample was then digested in a mixture of $3 \mathrm{U}$ DNase and 25 $\mathrm{U}$ RNase at $37^{\circ} \mathrm{C}$ for $90 \mathrm{~min}$ in $10 \times$ DNase buffer (NEB). The viral DNA and RNA were simultaneously extracted using a TaKaRa MiniBEST Viral RNA/DNA Extraction Kit Ver.5.0 (TaKaRa Inc., Japan).

\section{Reverse transcription (RT) and sequence-independent PCR amplification of viral nucleic acids}

Reverse transcription was performed using a Transcriptor First-Strand cDNA Synthesis Kit 6.0 (Roche Inc., USA) and 100 pmol of primer K-8N (GACCATCTAGCGACC TCCACNNNNNNNN) [21]. The first-strand cDNA of each sample was converted into double-stranded cDNA in the 
presence of $5 \mathrm{U}$ of Klenow fragment (NEB) in 10× NEB buffer 2 , which was incubated at $37{ }^{\circ} \mathrm{C}$ for $1 \mathrm{~h}$ and inactivated at $75{ }^{\circ} \mathrm{C}$ for $10 \mathrm{~min}$.

Sequence-independent PCR amplification was conducted with $5 \mu 1$ of double-stranded cDNA template in a final reaction volume of $50 \mu \mathrm{l}$, which contained $2 \times$ Gflex PCR Buffer, $200 \mu \mathrm{M}$ deoxynucleoside triphosphate (dNTP), $1 \mu \mathrm{M}$ primer K (GACCATCTAGCGACCTCCAC) and 1.25 U Tks Gflex DNA polymerase (TaKaRa). The PCR cycles were set as follows: $94^{\circ} \mathrm{C}$ for $1 \mathrm{~min}$, followed by 40 cycles of $98^{\circ} \mathrm{C}$ for $10 \mathrm{~s}, 55{ }^{\circ} \mathrm{C}$ for $30 \mathrm{~s}$ and $68^{\circ} \mathrm{C}$ for $1 \mathrm{~min}$.

\section{Library construction and sequencing}

A total of $1 \mu \mathrm{g}$ of DNA per sample was used as input material for DNA sample preparation. Sequencing libraries were generated using a NEBNext ${ }^{\circledR}$ Ultra ${ }^{\mathrm{TM}}$ DNA Library Prep Kit for Illumina (NEB) following the manufacturer's recommendations. Briefly, the DNA sample was fragmented by sonication to a size of $\sim 300 \mathrm{bp}$, and fragments were endpolished, poly-A tailed and ligated with full-length adaptors for Illumina sequencing by further PCR amplification. Finally, PCR products were purified (AMPure XP system, USA), and libraries were analyzed for size distribution using an Agilent 2100 Bioanalyzer (USA) and quantified using real-time PCR.

\section{Pre-processing sequence filter}

A pre-processing workflow was applied to ensure that highquality reads were obtained. The following reads were removed: (1) reads containing low-quality bases (Sanger quality value $\leqq 5$ and low-quality bases exceeding $40 \%$ of the length of the reads); (2) reads with excessive $\mathrm{N}$ bases ( $\geqq 10 \%$ of given length of the reads); (3) reads of less than $50 \%$ of a given length; (4) reads containing an overlap of $15 \mathrm{bp}$ with sequences on a user-defined primer/adaptor sequence list; (5) homopolymer-containing reads; and (6) duplicated reads.

The most closely related host genome sequences in this study were scanned and discarded with SOAP2 mapper software [22], by comparison with the available genome sequences of Myotis brandtii, Myotis davidii, Myotis lucifugus and Eptesicus fuscus (wholegenome shotgun sequencing project accession numbers: ANKR00000000.1, ANKR00000000.1, AAPE00000000.2 and ALEH00000000.1). Sequences originating from the host genome ( $\geqq 90 \%$ consistency) were deleted.

\section{Analysis of sequence reads and de novo metagenomic assembly}

Valid sequence reads were aligned with sequences in the NCBI non-redundant nucleotide database (NT), the non-redundant protein database (NR) and viral Refseq databases (downloaded from the NCBI FTP server in November 2014) using BLASTn, BLASTx and tBlastx, respectively. The BLAST hits were defined as significant when e-value $\leq 10^{-5}$ [14]. A rapid, highly restrictive BLASTn homology search (with word size 40) against a non-redundant nucleotide (nt) database was performed to eliminate additional host reads and eukaryotic contaminants. The virus-like clean data was then subjected to further analysis.

Clean data from the above filter process were assembled using SOAP de novo assembling software [23] using different K-mer references $(45,55,59)$. Contigs with the largest N50 were selected. Assembled sequence contigs greater than 150 bp were compared with sequences in the GenBank nonredundant nucleotide database using BLASTn. The reference sequence of the source organism with the best contig alignment, with an e-value cut-off of $10^{-5}$, was retrieved for further phylogenetic analysis. More than one hit was sometimes obtained for each unassembled read and contig, at different taxonomic levels. To guarantee biological significance, the eligible sequences were parsed and exported with MetaGenome Analyzer (MEGAN 4) using the LCA algorithm to assign each sequence to the appropriate taxon using the NCBI taxonomic database [24]. The first and best hit was regarded as the taxonomic annotation of the contigs or unassembled reads.

\section{Molecular epidemiology of bat PVs, RVA, CVs and PiVs}

To delineate the molecular epidemiology of bat PVs, RVA, $\mathrm{CVs}$ and PiVs from different bat species in four geographic regions of Guangdong and Hainan provinces in southern China, previously described PCR primers were used to amplify the conserved regions of the Late 1 (L1) gene of PVs (450 bp), the major capsid region (VP7) gene of RVA (847 bp), the RdRp gene of CVs (319-331 bp) and the 3Dpol gene of PiVs (571 bp), respectively.

\section{Phylogenetic analysis}

Sequence editing and identity calculations were conducted by using BioEdit, version 7.0.4 (http://www.mbio.ncsu.edu/ BioEdit/bioedit.html). Nucleotide and amino acid sequence alignments were constructed for different open reading frames (ORFs) and the corresponding amino acid alignment using Clustal W version 2.0 [25]. Phylogenetic trees were constructed using Clustal W version 2.0 and Mega 6 [26] according to the neighbor-joining and maximum-likelihood algorithms. Bootstrap values of the constructed phylogenetic trees were generated by iteration using 1,000 replicates. 


\section{Nucleotide sequence accession numbers}

All sequences in this study have been submitted to GenBank. The accession numbers for the pol gene of retrovirus, IVa2 gene of adenovirus (AdV), Vp7 gene of rotavirus and L1 gene of PV are KY321921, KY321918, KU746889KU746892 and KU727225-KU727235, respectively.

\section{Results}

\section{Bat sampling}

Bat samples were collected between August 2011 and October 2014 in four cities (Huizhou, Guangzhou, Yunfu and Haikou) in southern China. We sampled the fecal specimens from 500 bats ( 6 species). Eighty-six fecal samples, classified by species and collection site, were pooled as six samples (E-Table 2). These samples were processed as follows: nucleic acid purification, RNA/DNA extraction and sequence-independent RT-PCR for NGS. Molecular epidemiological analysis of of RVA, PV, CV and PiV in the 500 bats was also conducted (E-Table 3).

\section{Virome analysis}

\section{Viral metagenomic overview}

After removing the contaminating reads, 108,443,256 sequence reads with an average length of $150 \mathrm{bp}$ had been generated from the extracted nucleic acids (Table 1). The fecal samples from bats revealed significant microbial diversity. A total of 90,181,623 sequence reads were classified as cellular organisms, including bacteria, archaea, eukaryota and other unassigned reads. There were 18,261,633 reads that best matched to viral proteins. The sequences from each pool were assembled de novo into contigs of variable lengths. A total of $13,466,976$ sequences were assembled into viral contigs (average length: $182 \mathrm{bp}$, e-value $\leqq 0.0001$ ), among which 5,496 were viral contigs longer than $500 \mathrm{bp}$ (average length: $776 \mathrm{bp}$ ).

The total viral contigs could be assigned into five viral clades, including 54 families and 219 genera: Reverse-Transcribing Viruses, dsDNA Viruses, dsRNA Viruses, ssDNA Viruses and ssRNA Viruses. Vertebrate viruses comprised the largest population and included viruses classifiable as members of the Alloherpesviridae, Herpesviridae, Adenoviridae, Orthomyxoviridae, Papillomaviridae, Retroviridae, Hepadnaviridae, Flaviviridae, Polyomaviridae, Paramyxoviridae, Rhabdoviridae, Coronaviridae, Picornaviridae, Arenaviridae, Astroviridae, Bunyaviridae, Iridoviridae, Poxviridae and Circoviridae families (Figure 1, Table 2; please refer to Table 2 for details of other viral contigs, plant, bacterial, fungal, archaeal and algal in nature).

There were 1424 deltaretrovirus-related contigs, accounting for most of the virus-related contigs. These had the highest amino acid identity with the Pol protein of Pteropus vampyrus endogenous retrovirus group K (90\%). Sequences of contigs from members classifiable within the genera Phlebovirus, Tospovirus, Rotavirus A, Mastarenavirus, Picobirnavirus, Simplexvirus, Varicellovirus, Mastadenovirus, Circovirus, Mamastrovirus, Coronavirus, Orthohepadnavirus, Reoviridae, Iflavirus, Bracovirus, Orthopoxvirus, Vesiculovirus and Chrysovirus (as well as all phage-like contigs) shared an identity of greater than $80 \%$ with sequences in the existing database. In addition, the contigs representing influenza A viruses (with identities of $89.6-100 \%$ to sequences in the database, average: $92.8 \% ; 13.2 \%$ of the sequence identities were greater than $95 \%$ ) and coronaviruses (identities of $95-100 \%$, average: $99.8 \%$ ) found in bats shared high
Table 1 Overview of Solexa sequencing

\begin{tabular}{lrrrrrrr}
\hline Pool & Data size & Viral Reads & Others & \multicolumn{1}{l}{ Contigs } & A.length & Contigs $>500 \mathrm{bp}$ & $\begin{array}{l}\text { A.length } \\
>500 \mathrm{bp}\end{array}$ \\
\hline GZ.CS & $10,337,998$ & $5,442,833$ & $4,895,165$ & $5,081,665$ & 168 & 386 & 861 \\
HN.MS & $15,584,606$ & $8,015,941$ & $7,568,665$ & $6,742,580$ & 197 & 1176 & 783 \\
HN.RL & $35,722,372$ & 244,671 & $35,477,701$ & 80,568 & 209 & 805 & 650 \\
HZ.HL & $22,860,172$ & 337,084 & $22,523,088$ & 107,376 & 201 & 1267 & 847 \\
YF.RB & $8,191,142$ & $2,580,806$ & $5,610,336$ & $1,021,724$ & 162 & 1232 & 829 \\
YF.SK & $15,746,966$ & $1,640,298$ & $14,106,668$ & 433,066 & 156 & 630 & 687 \\
Total & $108,443,256$ & $18,261,633$ & $90,181,623$ & $13,466,976$ & 182 & 5496 & 776 \\
\hline
\end{tabular}

GZ.CS: Cynopterus sphinx collected in Guangzhou; HN.MS: Miniopterus schreibersi collected in Hainan; HN.RL: Rousettus leschenaulti collected in Hainan; HZ.HL: Hipposideros larvatus collected in Huizhou; YF.RB: Rhinolophus blythi collected in Yunfu; YF.SK: Scotophilus kuhlii collected in Yunfu; Data size: the total reads after removal of contamination by host genome; Reads: the total length of viral reads; Contigs: the total length of contigs after overlapping; A. length: the average length of contigs; A. Length $>500 \mathrm{bp}$ : the average length of contigs $>500 \mathrm{bp}$; Others: the total length of reads from eukaryotic, bacterial and unknown sequences without a hit in the GenBank non-redundant database 

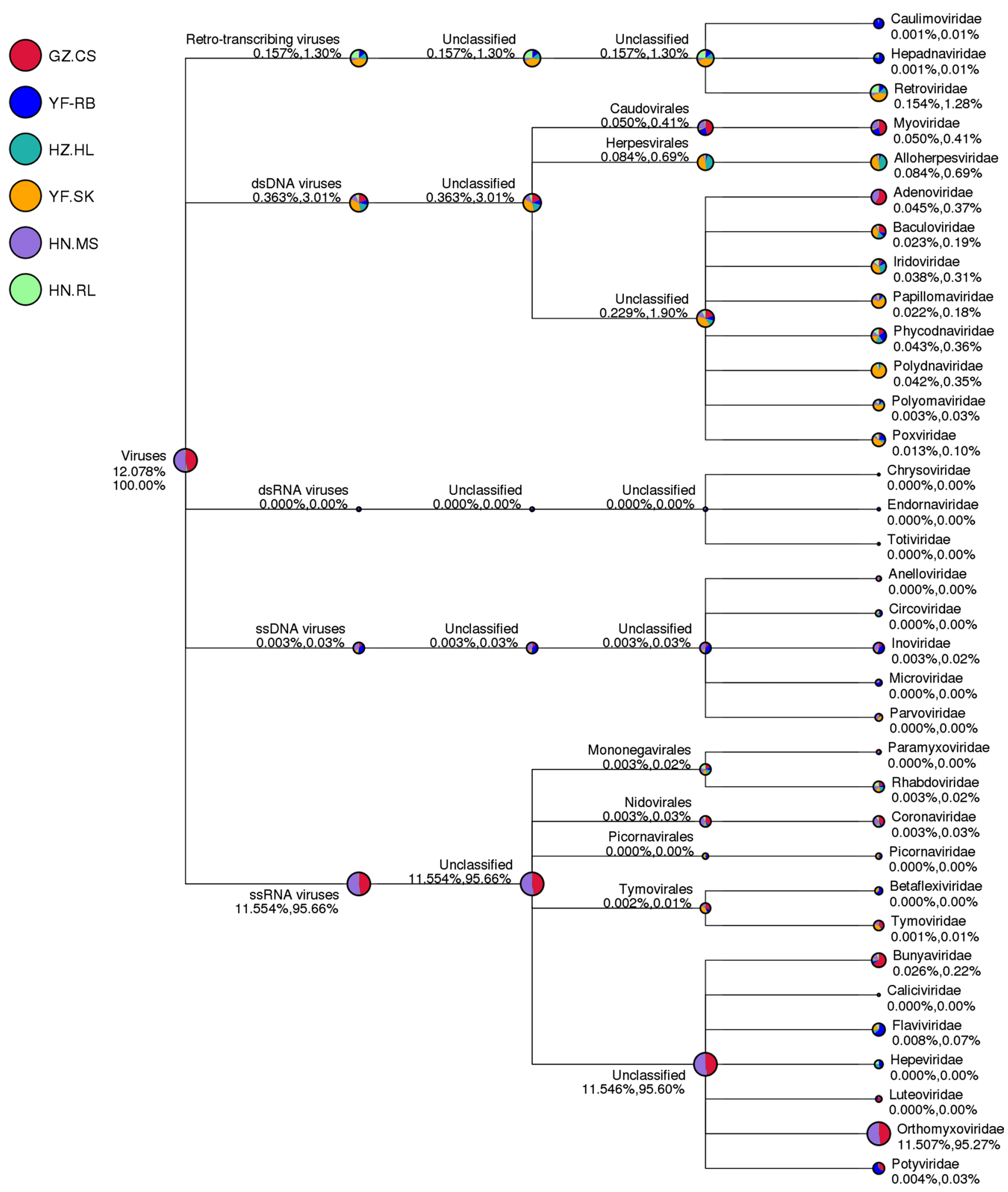

Fig. 1 Taxonomy information for the six bat groups using the MEGAN software. GZ.CS: Cynopterus sphinx collected in Guangzhou; HN.MS: Miniopterus schreibersi collected in Hainan; HN.RL:
Rousettus leschenaulti collected in Hainan; HZ.HL: Hipposideros larvatus collected in Huizhou; YF.RB: Rhinolophus blythi collected in Yunfu; YF.SK: Scotophilus kuhlii collected in Yunfu 
Table 2 Overview of the viral contigs identified in this study

\begin{tabular}{|c|c|c|c|c|c|c|c|c|c|}
\hline Virus host & Viral family & Viral genus & HN.MS & GZ.CS & YF.RB & YF.SK & HZ.HL & HN.RL & Total \\
\hline \multirow[t]{46}{*}{ Vertebrate } & \multirow[t]{2}{*}{ Alloherpesviridae } & Cyprinivirus & 6 & 0 & 9 & 3 & 16 & 18 & 52 \\
\hline & & Ictalurivirus & 0 & 0 & 0 & 6 & 15 & 63 & 84 \\
\hline & \multirow[t]{11}{*}{ Herpesviridae } & Cytomegalovirus & 15 & 3 & 15 & 12 & 16 & 11 & 69 \\
\hline & & Lymphocryptovirus & 12 & 0 & 6 & 9 & 4 & 5 & 36 \\
\hline & & Macavirus & 3 & 0 & 0 & 0 & 2 & 1 & 6 \\
\hline & & Mardivirus & 0 & 0 & 0 & 0 & 10 & 14 & 24 \\
\hline & & Muromegalovirus & 0 & 0 & 0 & 0 & 0 & 1 & 1 \\
\hline & & Percavirus & 0 & 0 & 0 & 0 & 0 & 2 & 2 \\
\hline & & Proboscivirus & 0 & 0 & 0 & 0 & 4 & 8 & 12 \\
\hline & & Rhadinovirus & 0 & 0 & 3 & 9 & 1 & 4 & 17 \\
\hline & & Roseovirus & 3 & 3 & 0 & 9 & 7 & 25 & 47 \\
\hline & & Simplexvirus & 0 & 0 & 3 & 9 & 2 & 13 & 27 \\
\hline & & Varicellovirus & 0 & 0 & 0 & 3 & 16 & 26 & 42 \\
\hline & Adenoviridae & Mastadenovirus & 15 & 24 & 0 & 0 & 1 & 1 & 41 \\
\hline & Orthomyxoviridae & Influenzavirus A & 3 & 3 & 78 & 0 & 162 & 254 & 500 \\
\hline & Papillomaviridae & Alphapapillomavirus & 15 & 3 & 15 & 8 & 142 & 118 & 301 \\
\hline & \multirow[t]{7}{*}{ Retroviridae } & Alpharetrovirus & 54 & 0 & 9 & 12 & 8 & 10 & 93 \\
\hline & & Gammaretrovirus & 105 & 5 & 21 & 0 & 23 & 78 & 232 \\
\hline & & Lentivirus & 15 & 0 & 3 & 6 & 21 & 78 & 123 \\
\hline & & Rotavirus & 0 & 0 & 12 & 9 & 4 & 18 & 43 \\
\hline & & Betaretrovirus & 0 & 0 & 9 & 15 & 16 & 44 & 84 \\
\hline & & Deltaretrovirus & 0 & 0 & 3 & 39 & 18 & 1364 & 1424 \\
\hline & & Unclassified & 0 & 25 & 48 & 120 & 16 & 66 & 275 \\
\hline & \multirow[t]{2}{*}{ Hepadnaviridae } & Orthohepadnavirus & 0 & 0 & 3 & 0 & 2 & 3 & 8 \\
\hline & & Hepevirus & 0 & 0 & 6 & 0 & 5 & 4 & 15 \\
\hline & \multirow[t]{2}{*}{ Flaviviridae } & Hepacivirus & 3 & 0 & 30 & 0 & 8 & 15 & 56 \\
\hline & & Pestivirus & 0 & 0 & 36 & 0 & 15 & 36 & 87 \\
\hline & Polyomaviridae & Polyomavirus & 3 & 0 & 0 & 3 & 15 & 45 & 66 \\
\hline & Paramyxoviridae & Respirovirus & 0 & 0 & 0 & 0 & 0 & 2 & 2 \\
\hline & \multirow[t]{2}{*}{ Rhabdoviridae } & Bracorhabdovirus & 0 & 0 & 0 & 0 & 0 & 6 & 6 \\
\hline & & Novirhabdovirus & 0 & 0 & 0 & 0 & 3 & 0 & 3 \\
\hline & Coronaviridae & Coronavirius & 0 & 0 & 0 & 0 & 0 & 1 & 1 \\
\hline & \multirow[t]{3}{*}{ Picornaviridae } & Aphthovirus & 0 & 0 & 0 & 0 & 0 & 3 & 3 \\
\hline & & Enterovirus & 0 & 0 & 0 & 3 & 1 & 1 & 5 \\
\hline & & Parechovirus & 0 & 0 & 3 & 0 & 0 & 0 & 3 \\
\hline & Arenaviridae & Arenavirus & 0 & 0 & 0 & 0 & 1 & 5 & 5 \\
\hline & Astroviridae & Mamastrovirus & 0 & 0 & 0 & 0 & 0 & 1 & 1 \\
\hline & \multirow[t]{3}{*}{ Bunyaviridae } & Hantavirus & 0 & 0 & 0 & 0 & 0 & 9 & 9 \\
\hline & & Orthobunyavirus & 0 & 0 & 3 & 0 & 1 & 12 & 16 \\
\hline & & Phlebovirus & 0 & 0 & 0 & 0 & 7 & 0 & 7 \\
\hline & Iridoviridae & Iridovirus & 0 & 0 & 0 & 0 & 0 & 4 & 4 \\
\hline & \multirow[t]{3}{*}{ Poxviridae } & Avipoxvirus & 0 & 0 & 0 & 0 & 0 & 1 & 1 \\
\hline & & Molluscipox virus & 0 & 0 & 3 & 0 & 4 & 9 & 16 \\
\hline & & Orthopoxvirus & 0 & 0 & 9 & 0 & 13 & 31 & 53 \\
\hline & \multirow[t]{2}{*}{ Circoviridae } & Circovirus & 0 & 0 & 3 & 0 & 0 & 0 & 3 \\
\hline & & Subtotal & 252 & 66 & 330 & 275 & 579 & 2410 & 3912 \\
\hline \multirow[t]{4}{*}{ Plant } & Luteoviridae & Polerovirus & 0 & 0 & 0 & 0 & 0 & 0 & 0 \\
\hline & Bromoviridae & Cucumovirus & 0 & 0 & 0 & 0 & 2 & 4 & 6 \\
\hline & Bunyaviridae & Tospovirus & 0 & 0 & 0 & 3 & 19 & 151 & 173 \\
\hline & Potyviridae & Potyvirus & 0 & 0 & 36 & 0 & 0 & 5 & 41 \\
\hline
\end{tabular}


Table 2 (continued)

\begin{tabular}{|c|c|c|c|c|c|c|c|c|c|}
\hline Virus host & Viral family & Viral genus & HN.MS & GZ.CS & YF.RB & YF.SK & HZ.HL & HN.RL & Total \\
\hline & & Subtotal & 0 & 0 & 36 & 3 & 21 & 160 & 220 \\
\hline \multirow[t]{3}{*}{ Fungi } & Chrysoviridae & Chrysovirus & 0 & 0 & 0 & 0 & 2 & 12 & 14 \\
\hline & Narnaviridae & Mitovirus & 0 & 0 & 0 & 6 & 1 & 2 & 9 \\
\hline & & Subtotal & 0 & 0 & 0 & 6 & 3 & 14 & 23 \\
\hline \multirow[t]{7}{*}{ Bacteria } & Siphoviridae & Lambdalikevirus & 15 & 0 & 24 & 0 & 0 & 0 & 39 \\
\hline & & Tunalikevirus & 0 & 0 & 0 & 30 & 0 & 1 & 31 \\
\hline & & Tm4likevirus & 0 & 0 & 0 & 0 & 1 & 1 & 2 \\
\hline & Myoviridae & T4 like virus & 0 & 0 & 9 & 0 & 1 & 1 & 11 \\
\hline & & T7 likev irus & 0 & 0 & 3 & 36 & 0 & 1 & 40 \\
\hline & Bicaudaviridae & Bicaudavirus & 0 & 0 & 0 & 0 & 0 & 1 & 1 \\
\hline & & Subtotal & 15 & 0 & 36 & 66 & 2 & 5 & 124 \\
\hline \multirow[t]{7}{*}{ Insect } & Parvovirida & Iteradensovirus & 18 & 0 & 0 & 15 & 0 & 0 & 33 \\
\hline & Potyviridae & Potyviridae & 0 & 0 & 0 & 0 & 0 & 4 & 4 \\
\hline & Baculoviridae & Alphabaculovirus & 0 & 0 & 0 & 0 & 9 & 12 & 21 \\
\hline & & Betabaculovirus & 0 & 0 & 0 & 0 & 1 & 4 & 5 \\
\hline & Polydnaviridae & Bracovirus & 0 & 0 & 3 & 6 & 53 & 96 & 158 \\
\hline & & Ichnovirus & 0 & 0 & 0 & 0 & 8 & 22 & 30 \\
\hline & & Subtotal & 18 & 0 & 3 & 21 & 71 & 138 & 251 \\
\hline \multirow[t]{2}{*}{ Archaea } & Rudiviridae & Rudiviridae & 0 & 0 & 0 & 0 & 0 & 2 & 2 \\
\hline & & Subtotal & 0 & 0 & 0 & 0 & 0 & 2 & 2 \\
\hline \multirow[t]{4}{*}{ Alga } & Phycodnaviridae & Chlorovirus & 0 & 0 & 3 & 0 & 1 & 12 & 16 \\
\hline & & Coccolithovirus & 0 & 0 & 0 & 0 & 4 & 0 & 4 \\
\hline & & Subtotal & 0 & 0 & 3 & 0 & 5 & 14 & 22 \\
\hline & & Total & 285 & 66 & 408 & 371 & 681 & 2741 & 4552 \\
\hline
\end{tabular}

sequence identity with those from humans. Other contigs including those classifiable as members of the genera Alphapapillomavirus (sequence identities of 82.8-92.5\%, average: $83.4 \%$ ), Betaretrovirus (61.9-84.3\%, average: 69.7\%), Alpharetrovirus (46.5-86.1\%, average: 67.5\%), Varicellovirus (28.7-100\%, average: 49.7\%), Cyprinivirus (35.6-100\%, average: 58.5\%), Chlorovirus (33.3-77.8\%, average: $53.4 \%$ ) and Cucumovirus (46.9-68.8\%, average: $57.1 \%$ ) had low sequence identity to viruses in the existing databases (data not shown).

\section{Bat ReVs (retroviruses)}

A total of 2274 contigs were identified as belonging to the five genera of retroviruses (alpha-, beta-, gamma-, deltaand unclassified retroviruses), with e-values of less than $10^{-5}$ (Table 2). The retroviral sequences identified herein represented two genes, encoding the protease/polymerase (Pol) and envelope glycoproteins (Env). According to the BLASTx analysis, some of these genes were very closely related to those of Pteropus vampyrus endogenous retroviruses. All retroviral contigs that encoded the Env protein also contained stop codons within the translated region, according to BLASTx analysis. One of the longest retrovirus coding sequences encoding a Pol protein was used to construct a phylogenetic tree (Figure 2). The phylogenetic trees indicated that the longest Pol (HN.RL, accession number: KY321921) sequence identified clustered with those of Pteropus vampyrus endogenous retroviruses. HN.RL shared the highest amino acid sequence identity $(85.7 \%)$ with Pteropus vampyrus endogenous retroviruses, when compared to other retroviruses detected in bats (56.7-59.8\% sequence identity), duck (57.4\%), fowl (56.7\%), galidia (62.1\%), murine (57.7-60.1\%), baboon (58.1\%), koala (58.1\%) and porcine $(57.4 \%)$ sources (Table 3 ).

\section{Bat AdVs (adenoviruses)}

We identified 41 contigs representing AdV genomes in Cynopterus sphinx (GZ.CS), Miniopterus schreibersii (HN.MS), Hipposideros larvatus (HZ.HL) and Rousettus leschenaultii (HN.RL). No AdV contigs were found in Scotophilus kuhlii (YF.SK) or Rhinolophus blythi (YF.RB). These AdV contigs represented 25 E4orf1 protein-encoding and 16 IVa2 mature protein-encoding genes. A phylogenetic tree based on the IVa2 gene sequences (Figure 3 ) revealed that the contig representing AdV in HN.MS fell within the lineage of human adenovirus $C$, sharing a sequence identity 
Fig. 2 Phylogenetic analysis of the pol gene of ReVs detected in bats from southern China, based on a partial 171 amino acid sequence. The tree was generated using the neighbor-joining algorithm and the $\mathrm{p}$-distance model. A bootstrap test of 1000 replicates was used. Numbers above the branches indicate $\mathrm{NJ}$ bootstrap values. Bold triangles indicate retroviruses detected in the present study. RL: Rousettus leschenaultii; HN: Hainan province

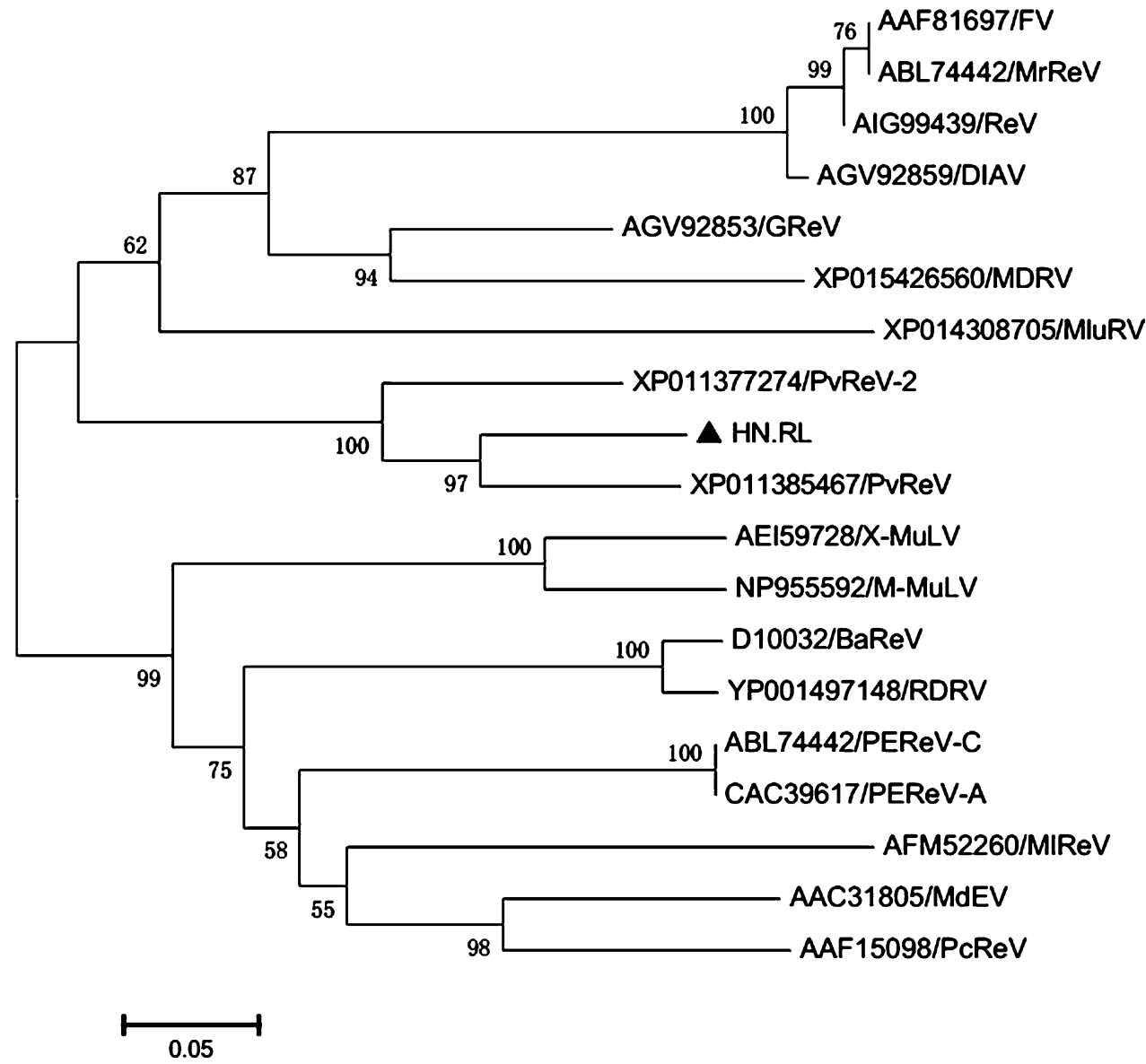

Table 3 Amino acid sequence identities (\%) comparing the partial POL gene between bat $\mathrm{ReVs}$ and other established $\mathrm{ReVs}$

\begin{tabular}{llll}
\hline ReVs in this study & Accession number & Host & Identity \\
\hline XP_011385467/PvReV & XP_011385467 & Pteropus vampyrus & 85.7 \\
XP_014308705/MluReV & XP_014308705 & Myotis lucifugus & 59.8 \\
AFM52260/MlyReV & AFM52260 & Megaderma lyra & 56.7 \\
XP_015426560/MdReV & XP_015426560 & Myotis davidii & 59.4 \\
ABL74442/MrReV & ABL74442 & Myotis ricketti & 56.7 \\
AGV92859/DIAV & AGV92859 & Duck & 57.4 \\
AAF81697/FV & AAF81697 & Fowl & 56.7 \\
AGV92853/GReV & AGV92853 & Galidia & 62.1 \\
NP_955592/M-MuLV & NP_955592 & Murine & 60.1 \\
AEI59728/X-MuLV & NP_040333 & Murine & 58.1 \\
D10032/BaReV & D10032 & Baboon & 58.1 \\
AAF15098/PcReV & AAF15098 & Phascolarctos cinereus & 58.1 \\
AAC31805/MdEV & AAC31805 & Mus dunni & 57.4 \\
CAC39617/PEReV-A & CAC39617 & Porcine & 57.4 \\
ABL74442/PEReV-C & ABL74442 & Porcine & 57.4 \\
\hline
\end{tabular}

PvRe: Pteropus vampyrus endogenous retrovirus; MluReV: Myotis lucifugus retrovirus;MlyReV: Megaderma lyra retrovirus; MrReV: Myotis ricketti retrovirus; MdReV: Myotis davidii retrovirus; DIAV: Duck infectious anemia virus; FV: Fowlpox virus; GReV: Galidia retrovirus; X-MuLV: Xenotropic murine leukemia virus; M-MuLV: Moloney murine leukemia virus; BaReV: Baboon endogenous retrovirus; PcReV: Phascolarctos cinereus retrovirus;PEReV-A: Porcine endogenous type A; PEReV-C: Porcine endogenous type $\mathrm{C}$ retrovirus class $\mathrm{C}$; MdEV: Mus dunni endogenous virus 
Fig. 3 Phylogenetic analysis of the IVa2 gene of AdVs detected in bats form southern China, based on a 102 amino acid sequence. The tree was generated using the neighbor-joining algorithm and the p-distance model. A bootstrap test of 1000 replicates was used. Numbers above the branches indicate $\mathrm{NJ}$ bootstrap values. Bold triangles indicate adenoviruses detected in the present study. MS: Miniopterus schreibersi; HN: Hainan province

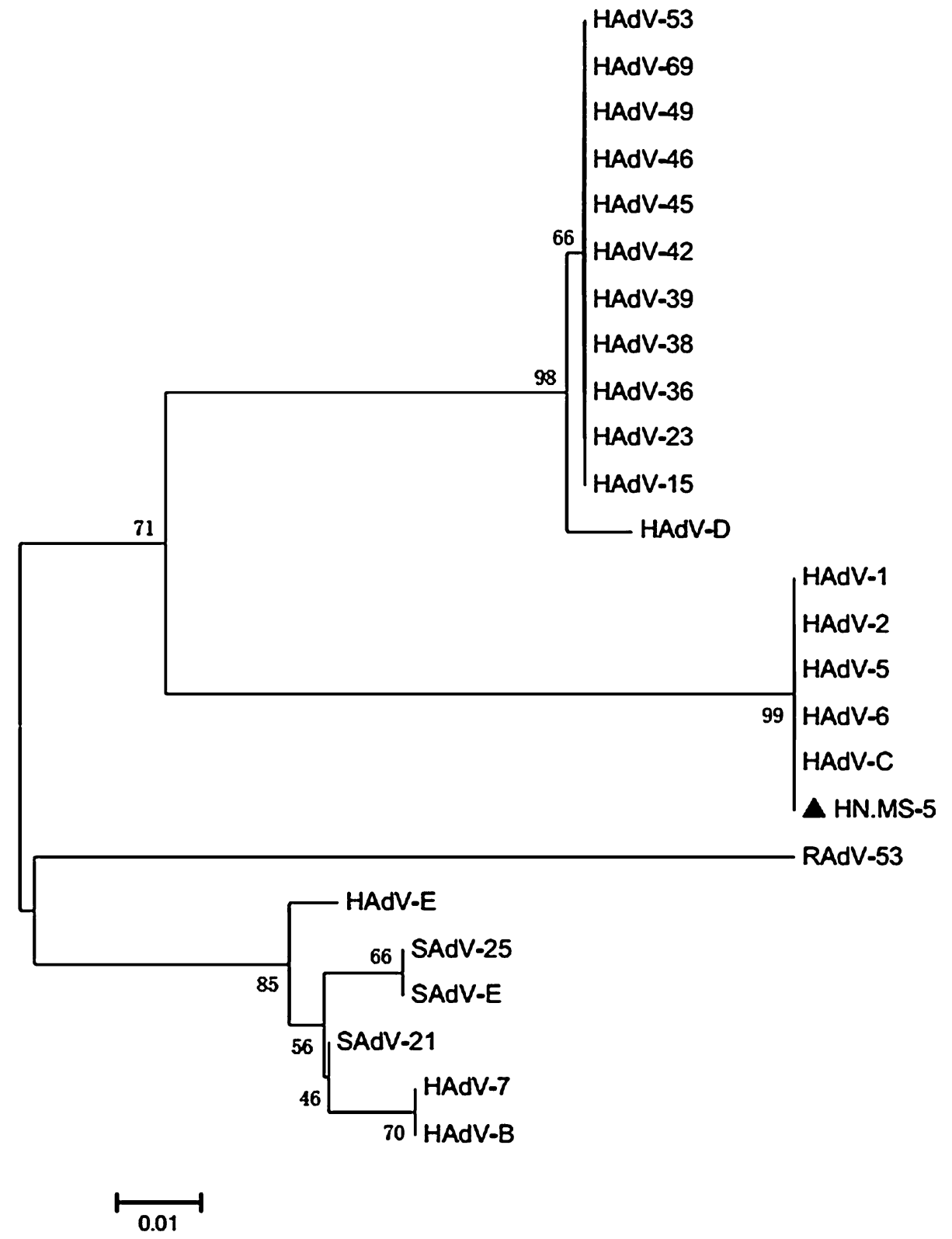

of $100 \%$. Additionally, HN.MS shared a sequence identity of $87.2-100 \%$ with human AdVs, compared with only 60.7-62.7\% sequence identity with bat AdVs (Table 4). This indicates the putative evolution of a novel viral strain.

\section{Prevalence and phylogenetic analysis of bat PVs (papillomaviruses)}

In total, $2.2 \%(11 / 500)$ of fecal samples tested positive for PVs, of which two were found in Scotophilus kuhlii and nine in Rhinolophus blythi (E-Table 3).

A phylogenetic tree was constructed based on an L1 gene nucleotide sequence alignment of 47 PV types (representing multiple PV species/genera) in combination with the bat PVs detected herein. The phylogenetic tree constructed
Table 4 Amino acid sequence identities (\%) for the partial IVa2 gene between bat AdVs and other established AdVs

\begin{tabular}{llll}
\hline AdVs in this study & Accession number & Host & Identity \\
\hline HAdV-C & AGT75926 & Human & 100 \\
HAdV-6 & ACN88093 & Human & 100 \\
HAdV-D & AGT77812 & Human & 88.2 \\
HAdV-39 & AFK92646 & Human & 88.2 \\
HAdV-E & YP_068022 & Human & 87.2 \\
SAdV-E & AAS10359 & Simian & 87.2 \\
SAdV-21 & AP_000265 & Simian & 88.2 \\
HAdV-B & AFQ34416 & Human & 87.2 \\
RAdV-53 & YP_009110798 & Rhesus & 83.3 \\
BAdV-TJM & YP_005271181 & Bat & 62.7 \\
BAdV-2 & YP_004782099 & Bat & 60.7 \\
\hline
\end{tabular}


Fig. 4 Phylogenetic analysis of the L1 gene of papillomavirus detected in bats from southern China, based on a 392 nucleotide sequence. The phylogenetic tree was generated using the neighborjoining algorithm with the p-distance or Maximum Composite Likelihood model. A bootstrap test of 1000 replicates was used. The numbers above the branches indicate the NJ bootstrap values. Bold triangles indicate the papillomavirus detected in this study. Bold squares indicated the papillomavirus detected in bats. RB: Rhinolophus blythi; SK: Scotophilus kuhlii; YF: Yunfu; HZ: Huizhou

by the neighbor-joining algorithm clustered the established PVs into 17 distinct, previously defined genera, ranging from Alphapapillomavirus to Sigmapapillomavirus [27, 28]. Within this phylogenetic tree, the 11 bat PVs from Scotophilus kuhlii and Rhinolophus blythi clustered in the same clade, constituting a monophyletic clade with viruses classified within the genus Betapapillomavirus. However, the bat PVs reported here actually appear to belong to an unassigned genus because the bootstrap support value for the closest node was less than $80 \%$ (Figure 4). Eleven bat $\mathrm{PVs}$ shared high sequence similarities in the $\mathrm{L} 1$ gene region. Notably, the similarities of the PVs from Scotophilus kuhlii and Rhinolophus blythi ranged from $94.5 \%$ to $100 \%$. Likewise, low sequence similarity was found between the PVs reported here and previously detected bat PVs Ms-PVs-1 (42.1-43.6\% similarity), MrPVs1 (45.8-47.3\%) and RaPVs1 (47.7-50.0\%). Even when compared to the most closely related clade (Betapapillomavirus), the bat PVs shared a sequence similarity of only 55.0-58.9\%. The similarities, calculated by pairwise alignment of the corresponding amino acids, are provided in Table 5.

\section{Prevalence and phylogenetic analysis of bat RVA (rotavirus A)}

In total, $0.8 \%(4 / 500)$ of fecal samples tested positive for RVA strains, all of which were found in Scotophilus kuhlii (E-Table 3).

A phylogenetic tree was constructed based on a VP7 gene nucleotide sequence alignment of established genotypes, calculated using the neighbor-joining algorithm with the p-distance or Maximum Composite Likelihood algorithm. The four RVA strains detected herein clustered with mammalian RVAs, constituting a monophyletic clade among the genotype 3 strains; however, the bootstrap value of the node was only $50 \%$, which failed to confirm whether the bat RVAs detected herein belong to G3 (Figure 5).

The putative VP7 genes of the four RVA strains detected in Scotophilus kuhlii were 847 bp in length and encoded a 282 amino acid protein. When compared with other mammalian rotaviruses, the VP7 nucleotide sequences of the four Scotophilus kuhlii isolates showed low levels of sequence identity to the established G3 genotypes (58.0-80.2\%). Compared with human, bat, cat, dog

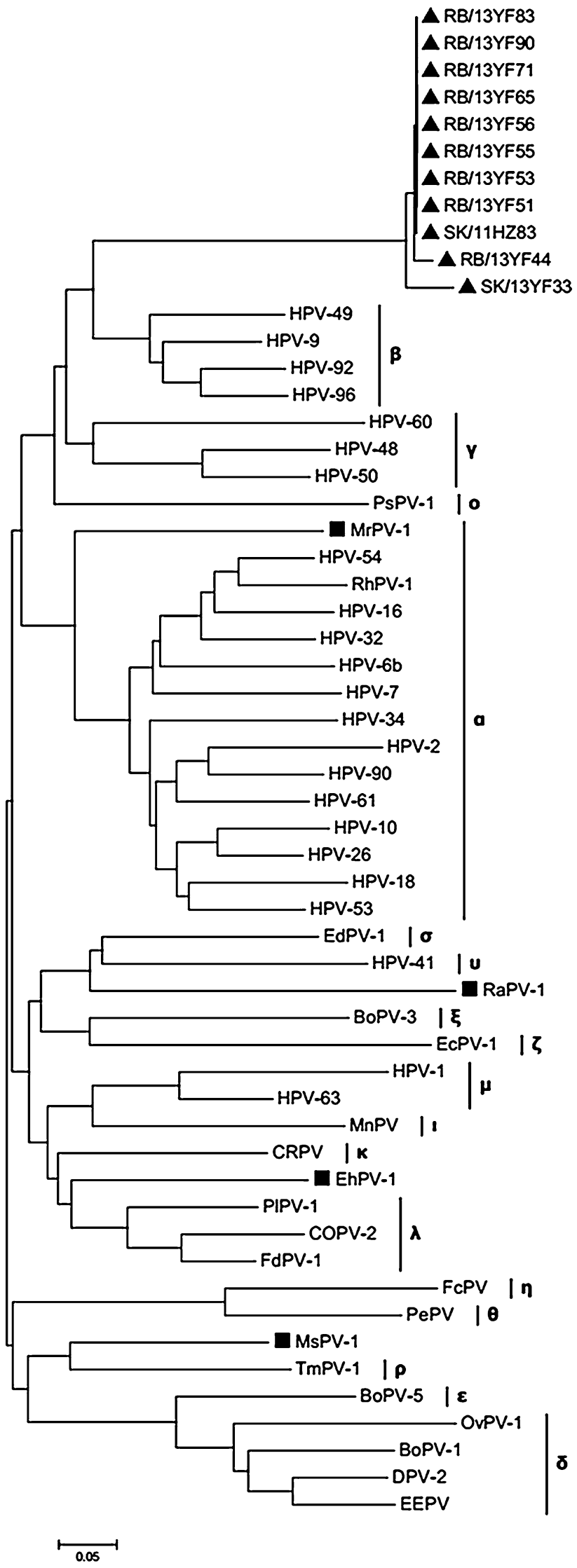


Table 5 Amino acid sequence identities (\%) for the partial L1 gene between bat papillomaviruses identified in this study and other previously reported papillomaviruses

\begin{tabular}{lllllllll}
\hline PV in this study & \multicolumn{3}{l}{ Betapalpillomavirus } & & & \multicolumn{3}{c}{ Gammapapillomavirus } \\
\cline { 2 - 3 } & HPV-9 & HPV-49 & HPV-92 & HPV-96 & & HPV-60 & HPV-48 & HPV-50 \\
\hline SK/11HZ83 & 57.3 & 57.3 & 58.9 & 58.1 & & 52.2 & 53.8 & 56.1 \\
RB/13YF51 & 57.3 & 57.3 & 58.9 & 58.1 & & 52.2 & 53.8 & 56.1 \\
RB/13YF44 & 56.5 & 56.5 & 57.3 & 57.3 & & 51.5 & 52.3 & 55.3 \\
RB/13YF53 & 57.3 & 57.3 & 58.9 & 58.1 & & 52.2 & 53.8 & 56.1 \\
RB/13YF55 & 57.3 & 57.3 & 58.9 & 58.1 & & 52.2 & 53.8 & 56.1 \\
RB/13YF56 & 57.3 & 57.3 & 58.9 & 58.1 & & 52.2 & 53.8 & 56.1 \\
RB/13YF65 & 57.3 & 57.3 & 58.9 & 58.1 & & 52.2 & 53.8 & 56.1 \\
RB/13YF71 & 57.3 & 57.3 & 58.9 & 58.1 & & 52.2 & 53.8 & 56.1 \\
RB/13YF83 & 57.3 & 57.3 & 58.9 & 58.1 & & 52.2 & 53.8 & 56.1 \\
RB/13YF90 & 57.3 & 57.3 & 58.9 & 58.1 & & 52.2 & 53.8 & 56.1 \\
SK/13YF33 & 55.0 & 55.0 & 56.5 & 55.8 & & 50.7 & 52.3 & 54.6 \\
\hline
\end{tabular}

and simian G3 RVA strains, the bat RVAs reported herein had sequence identities of $77.1-79.5 \%, 79.4-79.6 \%$, $79.3-79.4 \%, 78.3-80.2 \%$ and $77.6-80.0 \%$, respectively (Table 6).

The bat VP7 sequences shared a maximum nucleotide identity of $80.0 \%$ with that of a simian RVA strain (RVA_ simian-tc_USA_RRV_1975_G3P3), which is approaching the cut-off value $(80.0 \%)$ for VP7 classification proposed by the Rotavirus Classification Working Group (RCWG). The amino acid identities of the VP7 antigenic regions: A (87-100), B (141-150) and C (208-224) within these bat RVAs, when compared with the G3 types was 85.7-92.8\%, $80.0-100.0 \%$ and $82.3-88.2 \%$, respectively, whereas these sequences exhibited identities of 50.5-85.7\%, 50.0-100.0\% and $64.7-88.2 \%$ to other $\mathrm{G}$ types, respectively (Table 6).

\section{Prevalence and phylogenetic analysis of bat CVs and PiVs}

No CVs or PiVs were found in the 500 fecal specimens studied.

\section{Discussion}

To determine the potential for zoonotic virus transmission from bats to humans, we collected bat samples from habitats mainly in residential areas, city parks, abandoned houses and mine caves, all of which were close to the living environment of humans. This study examined the virome composition and viral abundance in fecal and rectal samples from six bat species collected in four cities in southern China. This represents the first time, to our knowledge, a report on the viral diversity of Cynopterus sphinx, Rousettus leschenaultii,
Hipposideros larvatus and Rhinolophus blythi in southern China.

\section{Virome analysis}

Similarly to previous studies [5-8], a considerable number of viral sequence reads could be classified into virus families: plant, fungal, bacterial, insect, archaeal and algal in nature. The identification of insect viruses, fungal viruses and plant viruses presumably corresponds to the habits and habitats of the bats, including insect-eating, cave-dwelling and social stucture. In addition, the identification of phages likely reflects the bacterial flora harbored inside the bats. Since we focused on the putative role of bats in zoonotic disease transmission, only mammalian virus contigs were analyzed further. Similarly to previous studies, most mammalian viruses were typical zoonotic viruses, including viruses classifiable as Influenza virus A, as well as members of the genera Mastadenovirus, Rotavirus, Mamastrovirus, Coronavirus, Hantavirus, Rhabdovirus and Parechovirus, and also members of the family Papillomaviridae. Metagenomic analyses in other studies have identified the full-length sequences of adenoviruses [8, 10, 12, 14], papillomaviruses [13, 14, 27, 28], parvoviruses [8, 13, 29], circoviruses [29], herpesviruses [11] and rotaviruses [12]. However, it is challenging to make specific or direct comparisons with our study due to significant methodological variations; for instance, the sample type (e.g., fresh guano, urine and roost guano), virus enrichment procedures (e.g., nuclease treatment, centrifugation and filtration) and amplification methods and/or high-throughput sequencing platforms [30]. The most important finding of our study was that the influenza $\mathrm{A}$ virus and coronavirus sequences we identified in bats shared their highest genetic identity with previous isolates from humans, suggesting possible cross-species transmission. However, contig sequences representing viruses classifiable 


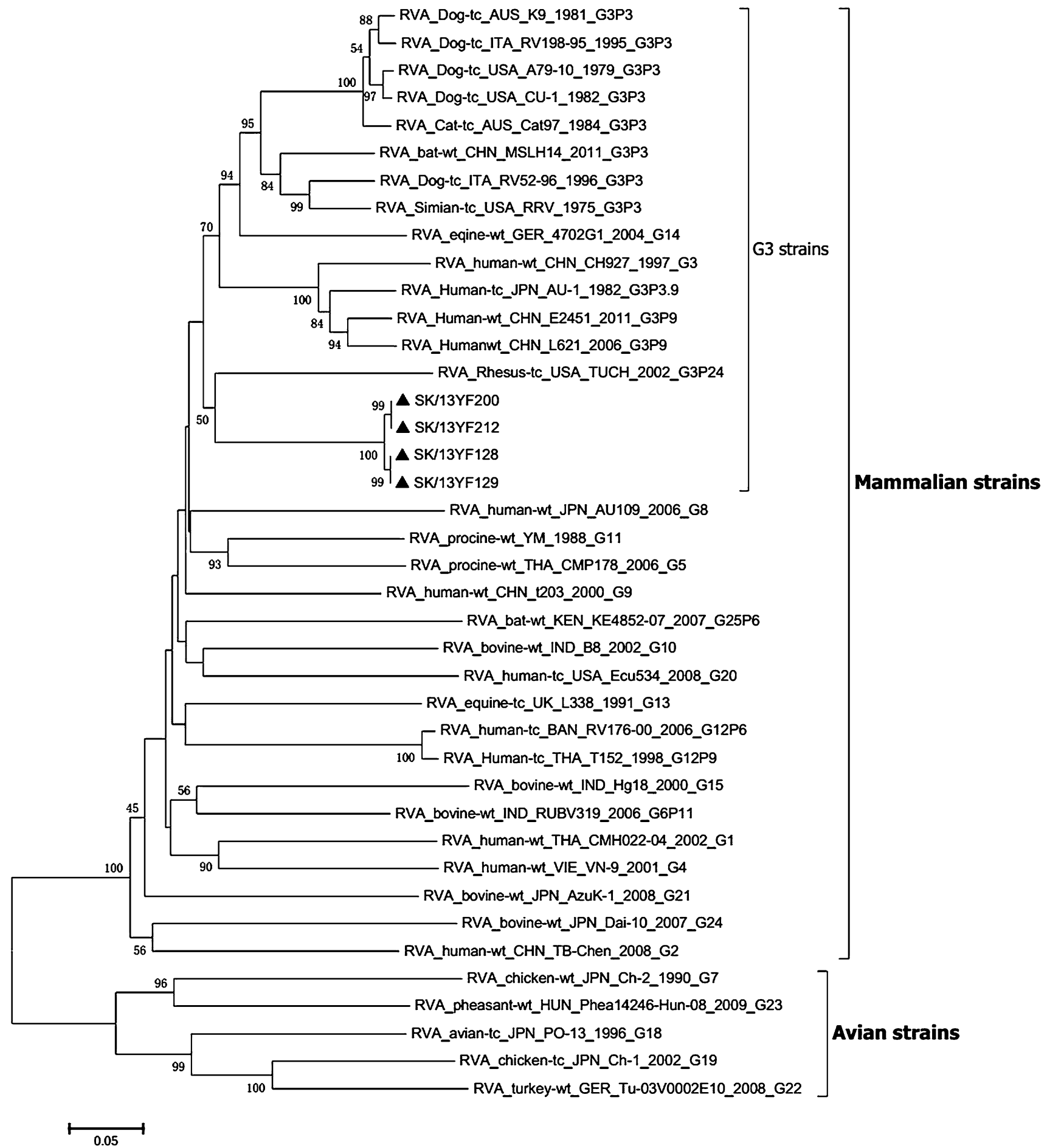

Fig. 5 Phylogenetic analysis of the major capsid region (vp7) of rotaviruses from bats in southern China, based on a 847 nucleotide sequence. The tree was generated using the neighbor-joining method with the p-distance or Maximum Composite Likelihood model. A bootstrap test of 1000 replicates was used. Numbers above the

within the genera Alphapapillomavirus, Betaretrovirus, Alpharetrovirus, Varicellovirus, Cyprinivirus, Chlorovirus and Cucumovirus were also identified that had identities branches indicate NJ bootstrap values. Bold triangles indicate rotaviruses detected in the present study. Abbreviations of virus names are shown in Figure 2, footnote. YF: Yunfu; SK: Scotophilus kuhlii; RB: Rhinolophus blythi

of less than $70 \%$ when compared with viruses in existing databases, indicating the possible evolution of novel viral strains. 
Table 6 Nucleotide identities (\%) of partial Vp7 gene sequences between bat rotaviruses and other established rotaviruses

\begin{tabular}{|c|c|c|c|c|}
\hline \multirow[t]{2}{*}{ RVA strains } & \multicolumn{4}{|c|}{ Genotype ( $\%$ nucleotide sequence identity) } \\
\hline & SK/13YF128 & SK/13YF129 & SK/13YF200 & SK/13YF212 \\
\hline RVA_human-wt_THA_CMH022-04_2002_G1 & 72.40 & 72.40 & 72.30 & 72.30 \\
\hline RVA_human-wt_CHN_TB-Chen_2008_G2 & 70.00 & 70.00 & 70.30 & 70.30 \\
\hline RVA_human-wt_CHN_CH927_1997_G3 & 77.10 & 77.10 & 77.20 & 77.20 \\
\hline RVA_bat-wt_CHN_MSLH14_2011_G3P3 & 79.60 & 79.60 & 79.40 & 79.40 \\
\hline RVA_cat-tc_AUS_Cat97_1984_G3P3 & 79.40 & 79.40 & 79.30 & 79.30 \\
\hline RVA_dog-tc_ITA_RV198-95_1995_G3P3 & 78.50 & 78.50 & 78.30 & 78.30 \\
\hline RVA_dog-tc_AUS_K9_1981_G3P3 & 78.30 & 78.30 & 78.30 & 78.30 \\
\hline RVA_simian-tc_USA_RRV_1975_G3P3 & 80.00 & 80.00 & 80.00 & 80.00 \\
\hline RVA_dog-tc_ITA_RV52-96_1996_G3P3 & 80.20 & 80.20 & 80.10 & 80.10 \\
\hline RVA_dog-tc_USA_A79-10_1979_G3P3 & 78.30 & 78.30 & 78.30 & 78.30 \\
\hline RVA_human-tc_JPN_AU-1_1982_G3P3.9 & 78.90 & 78.90 & 79.00 & 79.00 \\
\hline RVA_humanwt_CHN_L621_2006_G3P9 & 79.30 & 79.30 & 79.30 & 79.30 \\
\hline RVA_rhesus-tc_USA_TUCH_2002_G3P24 & 77.40 & 77.40 & 77.60 & 77.60 \\
\hline RVA_procine-wt_THA_CMP178_2006_G5 & 76.70 & 76.70 & 76.60 & 76.60 \\
\hline RVA_bovine-wt_IND_RUBV319_2006_G6P11 & 75.40 & 75.40 & 75.60 & 75.60 \\
\hline RVA_chicken-wt_JPN_Ch-2_1990_G7 & 59.60 & 59.60 & 59.90 & 59.90 \\
\hline RVA_human-wt_JPN_AU109_2006_G8 & 74.40 & 74.40 & 74.60 & 74.60 \\
\hline RVA_bovine-wt_IND_B8_2002_G10 & 72.30 & 72.30 & 72.20 & 72.20 \\
\hline RVA_procine-wt_YM_1988_G11 & 77.20 & 77.20 & 77.00 & 77.00 \\
\hline RVA_equine-tc_UK_L338_1991_G13 & 75.60 & 75.60 & 75.60 & 75.60 \\
\hline RVA_eqine-wt_GER_4702G1_2004_G14 & 77.20 & 77.20 & 77.10 & 77.10 \\
\hline RVA_bovine-wt_IND_Hg18_2000_G15 & 70.90 & 70.90 & 71.00 & 71.00 \\
\hline RVA_avian-tc_JPN_PO-13_1996_G18 & 63.10 & 63.10 & 63.10 & 63.10 \\
\hline RVA_chicken-tc_JPN_Ch-1_2002_G19 & 58.80 & 58.80 & 58.90 & 58.90 \\
\hline RVA_human-tc_USA_Ecu534_2008_G20 & 74.10 & 74.10 & 74.10 & 74.10 \\
\hline RVA_turkey-wt_GER_Tu-03V0002E10_2008_G22 & 58.00 & 58.00 & 58.10 & 58.10 \\
\hline RVA_pheasant-wt_HUN_Phea14246-Hun-08_2009_G23 & 61.60 & 61.60 & 61.50 & 61.50 \\
\hline RVA_bovine-wt_JPN_Dai-10_2007_G24 & 68.10 & 68.10 & 68.10 & 68.10 \\
\hline RVA_bat-wt_KEN_KE4852-07_2007_G25P6 & 73.50 & 73.50 & 73.50 & 73.50 \\
\hline
\end{tabular}

Similar to the findings of Yuan et al [15], retroviruses were one of the most commonly found viruses in bats. Rousettus leschenaultii from Hainan was found to harbor phylogenetically distant gammaretroviruses from those found in Myotis lucifugus, Megaderma lyra, Myotis davidii and Myotis ricketti, which suggested that bats might be suitable vectors for retrovirus transmission [31, 32]. Interestingly, we found that the AdV contigs from HN.RL (representing the IVa2 genes) were clustered with human adenovirus-C and shared higher amino acid sequence identity with human adenoviruses than previously reported bat AdVs. This might indicate the possible evolution of novel viral strains. Owing to the limited size and number of contigs generated by Illumina sequencing, conventional PCR assays are warranted to generate longer and more abundant sequences for further phylogenetic analysis [33, 34].

Comparable with previous studies [13, 15], except for retrovirus and $\mathrm{AdV}$, analysis of more in-depth virus sequencing information (including phylogeny and sequence identity) was challenging for the following reasons: 1) many reads could not be assembled into longer scaffolds because the NGS reads were randomly amplified throughout the whole viral genome; 2) incomplete DNase/ RNase enzyme digestion resulting in a lack of hits for a considerable number of sequence reads (including those from cellular organisms) during raw data analysis; 3) some viruses may have been present in the samples in relatively small amounts below the sensitivity of NGS detection; 4) host genome and other non-target nucleic acids could have lowered the sensitivity of high-throughput sequencing and introduced background noise into the results.

\section{Papillomaviruses}

The L1 ORF is characterized as the most conserved region in the PV genome and is used for PV classification. According 
to recent classification criteria: 1) PVs belong to different PV genera when they share less then $60 \%$ nucleotide sequence identity across the entire L1 ORF [28]; 2) the taxonomic status of PV types, subtypes and variants is based on the conventional criteria that the sequence of their L1 genes should be at least $10 \%, 2-10 \%$ and maximally $2 \%$ dissimilar from one another. Because the bat $\mathrm{L} 1$ sequences shared a maximum of $58.9 \%$ nucleotide identity with the L1 sequence of HPVs-92, these PVs cannot be placed in one of the existing genera. The bat PVs therefore represent tentative members of a novel, yet innominated, PV genus.

Our findings argue against the mainstream theory that PVs are highly species specific and co-evolve with their host. Compatible with the results of Garcia-Perezstrict et al [35], this hypothesis of virus-host co-evolution is rejected by the existence of 11 closely related bat PVs in Scotophilus kuhlii and Rhinolophus blythi and by the lack of congruence between bats and bat PV phylogenies. Second, the possible inter-transmission of PVs to infect two different bat species (Scotophilus kuhlii and Rhinolophus blythi) argues against strict host specificity. However, the L1 sequence detected herein was only $450 \mathrm{bp}$ in length, which does not represent the whole of the L1 gene. Further studies with additional genomic sequences are required to examine the potential pathogenic role of PVs in other bat species, which would provide insights into the ecology and evolution of PVs in bats. These findings might help address whether the prevailing paradigms regarding PV evolution should be reconsidered.

\section{Rotavirus}

The RVA VP7 glycoprotein ( $\mathrm{G}$ genotypes) elicits neutralizing antibodies, and, to date, $27 \mathrm{G}$ and $37 \mathrm{P}$ genotypes from various hosts have been identified [36]. The nearly fulllength VP7 sequences (847 nt) of four Scotophilus kuhlii rotavirus strains (SK/13YF128/129/200/212), encoding a 282-amino acid protein, were determined. These segments were most closely related to simian and dog RVA G3 strains (RVA_simian-tc_USA_RRV_1975_G3P3，RVA_dogtc_ITA_RV52-96_1996_G3P3), sharing a similarity of $80.0 \%$ and $80.2 \%$, respectively. Even though the similarity of these VP7 sequences did not exceed the appropriate cutoff $(80.0 \%)$, the VP7 sequences detected herein represented more than 500 nt of the ORF ( $>50 \%$ of the ORF). Hence, the four bat RVA strains reported herein might be tentative G3 strains according to the RCWG classification [37].

G3P [3] and G3P [9] are genotype combinations commonly found in canines (RV198, RV52, A79-10, K9, CU-1, AUS and ITA) [38, 39]. Previous reports have identified canine RVA strains that infect humans, including the strains Ro1845, HCR3A, AU-1, E2451, L621, T152, CMH222, PA260, PAH136, PAI58, 6212, CMH120 and CU-1 [38-41].
In addition, some isolated animal RVA strains are believed to have a distant ancestor in common with canine RVA strains, when taking into account the majority of their gene segments [41]. Two distinct simian RVA strains (RRV and TUCH) have multiple genotypes more typical of caninefeline rotaviruses [40], suggesting potential transmission of canine-like RVA strains to simians. Although the genetic relationship of VP7 sequences between the four bat RVA strains and ITA/RRV is relatively distant, they might share a common ancestor. In light of the fact that the four bat RVA strains are not closely related to any known RVA strain, we speculate that these viruses are true bat RVA strains rather than viruses transmitted from other species.

The positive detection rate of RVA strains was low, which might have arisen for the following reasons: 1) we collected samples from adult bats which were immunocompetent and can clear viruses rapidly; 2) no neonates were involved, whereas RVA strains are known to infect mainly infants and young animals. To better understand the prevalence of RVAs in bats, further molecular and serological investigations with larger sample sizes and more species of adult and neonatal bats in different geographical regions are required.

\section{Calicivirus and picornavirus}

Notably, CVs and PiVs were not detected in the present study. The detection rates of CVs and PiVs in bats was also low in previous studies. In 2014, Kemenesi et al [17] found only three novel CVs in 447 bat fecal specimens in Europe, segregating with the Hipposideros pomona sapovirus genus found in 2012 [16]. In another study only 12 picornaviruspositive respiratory and alimentary specimens were identified from 1108 bats and 18 species [18]. The lack of CVs and PiVs detected in our study may be accounted for by low carrying rates for these two viruses. Other causes might be variations in duration of the virus shedding in feces, or geographical/seasonal/bat species differences.

\section{Conclusion}

In conclusion, this study describes the virome of six bat species in southern China and provides a more comprehensive understanding of virus ecology in common bat species found in human habitats. The detection of PVs in Scotophilus kuhlii and Rhinolophus blythi argues against the hypothesis that PVs have strict host specificity and co-evolution. The four bat RVA strains we isolated might be tentative G3 strains, according to the RCWG classification.

Acknowledgements This work was supported by the National Natural Science Foundation of China (Grant no. 30972525). We thank Jianpeng Xiao for his insightful knowledge of the phylogenetic analysis. 
Author contributions The contribution of each author is outlined briefly here: XZ, MQ, JL, SC and SH performed experiments and analyzed data; XZ and JL contributed bioinformatics analysis; ZC and YW identified bat species; XZ, QC and WG conceived and designed the experiments and wrote the paper.

\section{Compliance with ethical standards}

Conflict of interest No competing financial interests exist.

\section{References}

1. Wilson DE, Reeder DM (2005) Mammal species of the world. A taxonomic and geographic reference, 3rd edn. Johns Hopkins University Press, Baltimore, pp 154-196

2. Lau SK, Woo PC, Lai KK, Huang Y, Yip CC, Shek CT et al (2011) Complete genome analysis of three novel picornaviruses from diverse bat species. J Virol 85:8819-8828

3. Li Y, Ge X, Zhang H, Zhou P, Zhu Y, Zhang Y et al (2010) Host range, prevalence, and genetic diversity of adenoviruses in bats. J Virol 84:3889-3897

4. Wibbelt G, Kurth A, Yasmum N, Bannert M, Nagel S, Nitsche A et al (2007) Discovery of herpesviruses in bats. J Gen Virol 88:2651-2655

5. Lau SK, Woo PC, Li KS, Huang Y, Tsoi HW, Wong BH et al (2005) Severe acute respiratory syndrome coronavirus-like virus in Chinese horseshoe bats. Proc Natl Acad Sci USA 102:14040-14045

6. Calisher CH, Childs JE, Field HE, Holmes KV, Schountz T (2006) Bats: important reservoir hosts of emerging viruses. Clin Microbiol Rev 19:531-545

7. Victoria JG, Kapoor A, Dupuis K, Schnurr DP, Delwart EL (2008) Rapid identification of known and new RNA viruses from animal tissues. PLoS Pathog 4(9):e1000163

8. Li L, Victoria JG, Wang C, Jones M, Fellers GM, Kunz TH et al (2010) Bat guano virome: predominance of dietary viruses from insects and plants plus novel mammalianviruses. J Virol 84(14):6955-6965

9. Donaldson EF, Haskew AN, Gates JE, Huynh J, Moore CJ, Frieman MB (2010) Metagenomic analysis of the viromes of three North American bat species: viral diversity among different bat species that share a common habitat. J Virol 84(24):13004-13018

10. Dacheux L, Cervantes-Gonzalez M, Guigon G, Thiberge JM, Vandenbogaert M, Maufrais C et al (2014) Preliminary study of viral metagenomics of French bat species in contact with humans: identification of new mammalian viruses. PLoS One 9(1):e87194

11. Wang J, Moore NE, Murray ZL, McInnes K, White DJ, Tompkins DM, Hall RJ (2015) Discovery of novel virus sequences in an isolated and threatened bat species, the New Zealand lesser shorttailed bat (Mystacina tuberculata). J Gen Virol 96(8):2442-2452

12. He B, Li Z, Yang F et al (2013) Virome profiling of bats from Myanmar by metagenomic analysis of tissue samples reveals more novel mammalian viruses. PLoS One 8(4):e61950

13. Ge X, Li Y, Yang X, Zhang H, Zhou P, Zhang Y, Shi Z (2012) Metagenomic analysis of viruses from bat fecal samples reveals many novel viruses in insectivorous bats in China. J Virol 86(8):4620-4630

14. Wu Z, Ren X, Yang L, Hu Y, Yang J, He G et al (2012) Virome analysis for identification of novel mammalian viruses in bat species from Chinese provinces. J Virol 86(20):10999-11012
15. Yuan L, Li M, Li L, Monagin C, Chmura AA, Schneider BS et al (2014) Evidence for retrovirus and paramyxovirus infection of multiple bat species in China. Viruses 6(5):2138-2154

16. Tse H, Tsang AK, Tsoi HW, Leung AS, Ho CC, Lau SK et al (2012) Identification of a novel bat papillomavirus by metagenomics. PLoS One 7(8):e43986

17. Kemenesi G, Dallos B, Görföl T et al (2014) Molecular survey of RNA viruses in Hungarian bats: discovering novel astroviruses, coronaviruses, and caliciviruses. Vector Borne Zoonotic Dis 14(12):846-855

18. Lau SK, Woo PC, Lai KK et al (2011) Complete genome analysis of three novel picornaviruses from diverse bat species. J Virol 85(17):8819-8828

19. He B, Yang F, Yang W, Zhang Y, Feng Y, Zhou J et al (2013) Characterization of a novel G3P[3] rotavirus isolated from a lesser horseshoe bat: a distant relative of feline/canine rotaviruses. J Virol 87(22):12357-12366

20. Linacre A, Lee JC (2005) Species determination: the role and use of the cytochrome b gene. Methods Mol Biol 297:45-52

21. Djikeng A, Halpin R, Kuzmickas R, Depasse J, Feldblyum J, Sengamalay $N$ et al (2008) Viral genome sequencing by random priming methods. BMC Genom 7(9):5

22. Li R, Yu C, Li Y, Lam TW, Yiu SM et al (2009) SOAP2: an improved ultrafast tool for short read alignment. Bioinformatics 25:1966-1967

23. Luo R, Liu B, Xie Y et al (2012) SOAPdenovo2: an empirically improved memory-efficient short-read de novo assembler. GigaScience 1(1): 18

24. Huson DH, Auch AF, Qi J, Schuster SC (2008) MEGAN analysis of metagenomic data. Genome Res 17:377-386

25. Larkin MA, Blackshields G, Brown NP, Chenna R, McGettigan PA, McWilliam H et al (2007) Clustal W and Clustal X version 2.0. Bioinformatics 23:2947-2948

26. Tamura K, Dudley J, Nei M, Kumar S (2007) MEGA4: molecular evolutionary genetics analysis (MEGA) software version 4.0. Mol Biol Evol 24:1596-1599

27. Rector A, Bossart GD, Ghim SJ, Sundberg JP, Jenson AB, Van Ranst M (2004) Characterization of a novel close-to-root papillomavirus from a Florida manatee by using multiply primed rollingcircle amplification: Trichechus manatus latirostris papillomavirus type 1. J Virol 78:12698-12702

28. de Villiers EM, Fauquet C, Broker TR, Bernard HU, zur Hausen $\mathrm{H}$ (2004) Classification of papillomaviruses. Virology 324:17-27

29. Lima FE, Cibulski SP, Dall Bello AG, Mayer FQ, Witt AA, Roehe $\mathrm{PM}$ et al (2015) A novel chiropteran circovirus genome recovered from a Brazilian insectivorous bat species. Genome Announc 25 3(6):e01393-15

30. Daly GM, Bexfield N, Heaney J et al (2011) A viral discovery methodology for clinical biopsy samples utilising massively parallel next generation sequencing. PLoS One 6:e28879

31. Chua KB, Crameri G, Hyatt A et al (2007) A previously unknown retrovirus of bat origin is associated with an acute respiratory disease in humans. Proc Natl Acad Sci USA 2104:11424-11429

32. Gifford RJ, Katzourakis A, Tristem M et al (2008) A transitional endogenous lentivirus from the genome of a basal primate and implications for lentivirus evolution. Proc Natl Acad Sci USA 105:20362-20367

33. Drexler JF, Corman VM, Muller MA et al (2012) Bats host major mammalian paramyxoviruses. Nat Commun 24(3):796

34. Baker KS, Leggett RM, Bexfield NH et al (2013) Metagenomic study of the viruses of African straw-colored fruit bats: detection of a chiropteran poxvirus and isolation of a novel adenovirus. Virology 441:95-106

35. Garcia-Perez R, Ibánez C, Godinez JM et al (2014) Novel papillomaviruses in free-ranging Iberian bats: no virus-host co-evolution, 
no strict host specificity, and hints for recombination. Genome Biol Evol 6(1):94-104

36. Matthijnssens J, Ciarlet M, McDonald SM et al (2011) Uniformity of rotavirus strain nomenclature proposed by the Rotavirus Classification Working Group (RCWG). Arch Virol 156:1397-1413

37. Matthijnssens J, Ciarlet M, Rahman M et al (2008) Recommendations for the classification of group A rotaviruses using all 11 genomic RNA segments. Arch Virol 153(8):1621-1629

38. Matthijnssens J, De Grazia S, Piessens J et al (2011) Multiple reassortment and interspecies transmission events contribute to the diversity of feline, canine and feline/canine-like human group A rotavirus strains. Infect Genet Evol 11:1396-1406
39. Tsugawa T, Hoshino Y (2008) Whole genome sequence and phylogenetic analyses reveal human rotavirus G3P[3] strains Ro1845 and HCR3A are examples of direct virion transmission of canine/ feline rotaviruses to humans. Virology 380:344-353

40. Wang YH, Pang BB, Zhou X et al (2013) Complex evolutionary patterns of two rare human $\mathrm{G} 3 \mathrm{P}[9]$ rotavirus strains possessing a feline/canine-like H6 genotype on an AU-1-like genotype constellation. Infect Genet Evol 16:103-112

41. Khamrin P, Maneekarn N, Peerakome S et al (2006) Molecular characterization of a rare $\mathrm{G} 3 \mathrm{P}[3]$ human rotavirus reassortant strain reveals evidence for multiple human-animal interspecies transmissions. J Med Virol 78:986-994 